\title{
Effects of basal insulin application on serum visfatin and adiponectin levels in type 2 diabetes
}

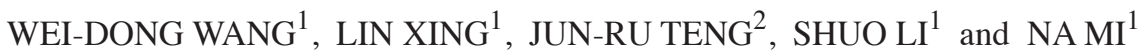 \\ Departments of ${ }^{1}$ Endocrinology and ${ }^{2}$ Clinical Laboratory, The Third People's Hospital of Jinan, \\ Jinan, Shandong 250132, P.R. China
}

Received March 17, 2014; Accepted October 28, 2014

DOI: $10.3892 /$ etm.2015.2428

\begin{abstract}
The aim of this study was to investigate the effects of basal insulin application on the serum visfatin and adiponectin (APN) levels of patients with type 2 diabetes mellitus (T2DM). A total of 200 patients with T2DM, who were diagnosed in The Third People's Hospital of Jinan (glycosylated hemoglobin $\geq 7 \%$ ), were randomly divided into treatment and control groups. The patients used only oral hypoglycemic drugs and had never received insulin therapy. In the treatment group, basal insulin was administered in combination with the original application of oral hypoglycemic drugs, whereas the control group maintained the original use of oral hypoglycemic drugs or took other oral hypoglycemic agents. The body mass index and fasting blood glucose, postprandial blood glucose, glycosylated hemoglobin, visfatin, APN and blood lipid levels of the patients were examined prior to the treatment and six months later. The drug and insulin doses in the treatment group were adjusted according to the patients' blood glucose, which allowed the fasting and postprandial blood glucose levels to attain the standards. The fasting and postprandial blood glucose levels in the control group also achieved the standards. It was found that the six-month application of basal insulin could significantly decrease the glycosylated hemoglobin and significantly increase the serum APN levels; the serum visfatin levels, however, remained unchanged. The immediate application of basal insulin could facilitate the attainment of glycosylated hemoglobin standards in T2DM and could increase the plasma APN levels, preventing diabetic vascular complications.
\end{abstract}

\section{Introduction}

In recent years, the worldwide prevalence of diabetes has shown a gradual annual increase. The prolonged history of

Correspondence to: Mr. Wei-Dong Wang, Department of Endocrinology, The Third People's Hospital of Jinan, 1 Wangsheren North Street, Gongye North Road, Jinan, Shandong 250132, P.R. China

E-mail: weidongwangen@163.com

Key words: basal insulin, type 2 diabetes, complications diabetes has demonstrated that the administration of a single oral antidiabetic drug (OAD) or combined OADs does not exhibit an ideal clinical controlling effect. Long-term hyperglycemia in patients with diabetes is associated with a lack of islet $\beta$-cell stimulation and activation. This condition leads to a functional depletion and a loss of sensitivity to glucose stimulation. Furthermore, long-term hyperglycemia may affect the transcription and translation of the insulin gene, reduce the synthesis and secretion of insulin (1), and increase the generation of oxygen free radicals within the $\beta$ cells. This occurrence results in the continuous opening of the voltage-dependent $\mathrm{Ca}^{2+}$ channel and the activation of the glucose-induced pro-apoptotic signaling pathway, ultimately inducing the apoptosis of the $\beta$ cells (1). The treatment consensus for type 2 diabetes mellitus (T2DM) published by the American Diabetes Association/European Diabetes Association (2) considers basal insulin therapy to be one of the preferred choices among the combined treatments for patients with T2DM who cannot achieve the standards for blood sugar after a three-month combined treatment of lifestyle changes and an OAD. A previous clinical study confirmed the efficacy and safety of the combined therapy of OAD and basal insulin (3).

The currently used forms of basal insulin are mainly recombinant neutral protamine hagedorn $(\mathrm{NPH})$ insulin, and analogues of long-acting insulin. For NPH insulin, zinc and protamine are added to human insulin to form a suspension, which can be injected subcutaneously, and is able to extend the time before insulin enters the peripheral blood circulation. Riddle et al (4) studied and compared the efficacies and safeties of glargine and NPH insulin when combined with oral hypoglycemic agents or pre-meal short-acting insulin in patients with T2DM. The results suggested that both glargine and NPH could achieve the same level of glycemic control, however the risk of hypoglycemia was lower with glargine, as compared with NPH insulin. Numerous clinical studies have demonstrated that the joint therapy of oral hypoglycemic agents and glargine could may significantly increase the standard-achieving rate of glycosylated hemoglobin (HbA1c $<7 \%$ ), and decrease the incidence of hypoglycemia, particularly nocturnal hypoglycemia $(5,6)$. Glargine is a recombinant human insulin analogue prepared using DNA recombinant technology; the aspartate, which is on the $21^{\text {st }}$ locus of the insulin A-chain, is replaced with glycine, and two arginines are added to the B-chain at the $30 \mathrm{a}$ and $30 \mathrm{~b}$ 
loci. Therefore, the intermolecular interactions are altered, and it forms micro-precipitates following subcutaneous injection, resulting in a steady release of insulin monomer, which may avoid the peak effect (4). In addition, insulin glargine has a pharmacodynamic time of $22 \pm 4 \mathrm{~h}$, with no obvious peak effect, similar to the effect curve of continuous insulin infusion (7). It has an action curve similar to continuous subcutaneous infusion of insulin (7). Accordingly, effective glycemic control throughout the day is achieved. Stentz et al (8) found that patients with severe hyperglycemia with associated elevated inflammatory mediators exhibited a significantly decreased level of inflammatory mediators following the insulin treatment. This result indicated that the insulin had a clinical anti-inflammatory effect. It has previously been demonstrated that adipocytes are an important source of proinflammatory cytokines. In addition, the endocrine dysfunction of adipocytes has been shown to have an important role in the inflammation-insulin resistance-diabetes pathological process (9). A previous study confirmed that adipose tissue is not only an energy storage organ, but also an endocrine organ, which may secrete various bioactive factors, such as visfatin, adiponectin and resistin, which are generally known as adipokines (10). Adipokines that are mainly produced by the visceral adipose tissue, such as visfatin, are involved in the occurrence and development of T2DM, obesity and atherosclerosis (11). A number of previous studies have suggested that adiponectin (APN) is closely associated with IR, which underlies the pathogenesis of T2DM $(12,13)$. APN is also considered to be an important biomarker of the body's IR level. APN-knockout mice exhibit severe IR (14); by contrast, intervention with recombinant APN can significantly improve the insulin sensitivity and hyperglycemia (15). A previous epidemiological study revealed that the serum APN levels of patients with diabetes were significantly and negatively correlated with insulin sensitivity (16). The mechanism underlying the regulation of blood sugar by APN was shown to be through the regulation of liver glucose metabolism and the inhibition of the production of endogenous hepatic glucose (17). APN can also affect the insulin signal transduction pathways in certain cells at the cellular level (18), indicating the existence of certain mechanisms allowing APN to exert its direct influence on the insulin signaling pathways. The APN levels in the serum of patients with diabetes have been shown to be significantly lower than those in normal individuals, and these low APN levels have exhibited clinical significance as indicators of the early findings of myocardial infarction and cardiac mortality (19).

The adipokine visfatin plays an important role in improving glucose tolerance and the occurrence of obesity-related IR and T2DM (20) and is highly expressed in the visceral fat. By phosphorylating the insulin receptor and receptor substrate, visfatin can cause protein kinase phosphorylation and specifically bind the insulin receptors to play an insulin-like role in the reduction of glucose levels and promoting the differentiation and synthesis of fat tissues. Increases in visfatin levels may act as a compensatory mechanism exhibited by the body when blood sugar levels are increased and $\beta$-cell functions are decreased. As such, visfatin may indirectly lead to IR, and increased levels of visfatin may be an indirect indicator of the severity of $\beta$-cell dysfunction. The blood sugar levels in patients with T2DM who are unable to obtain satisfactory control of their condition through the oral administration of hypoglycemic agents would be in a chronically high state (20). This long-term hyperglycemia could increase the oxidative stress and inflammation inside fat cells (21), as the dysfunction of islet $\beta$ cells and IR would result in the reduction of serum APN with the increase of visfatin.

Patients with diabetes who orally ingest hypoglycemic drugs but cannot attain the glycosylated hemoglobin standard should undergo basal insulin therapy as soon as possible in order to achieve strict control of their blood glucose and glycosylated protein levels, eliminate the toxicity of their high blood sugar and attenuate their IR. Accordingly, this would increase the serum APN and decrease the visfatin levels of the patients, and the macrovascular complications of diabetes would be prevented. There are currently no domestic nor international studies regarding the associations between insulin, APN and visfatin. The aim of the present study was therefore to examine patients with T2DM who could not achieve satisfactory results with OAD administration. The effects of basal insulin treatment on serum visfatin and APN levels were assessed, and the efficacy and safety of the treatment, with regard to the prevention of diabetes-related complications, were explored.

\section{Materials and methods}

Inclusion and exclusion criteria. Patient selection was performed based on the following inclusion criteria: i) age, 18-70 years; ii) firm diagnosis of T2DM; iii) treatment with OADs and iv) has received a glycosylated hemoglobin test during the past three months. During the enrollment diagnosis, the patient received a recommendation to undergo the basal insulin therapy. The patient had to agree to this therapy and voluntarily sign the informed consent. The exclusion criteria for the study population comprised patients who i) had been diagnosed with type 1 diabetes; ii) had used any type of insulin within the past two years (not including intermittent use for no more than one month each time); iii) had a serious disease that could lead to difficulties with the late follow-up appointment; and iv) were pregnant, breastfeeding or undergoing pregnancy planning. Any patients who were part of a clinical trial for other drugs or who had finished a trial in the previous month were also excluded from the study. This study was conducted in accordance with the Declaration of Helsinki and with approval from the Ethics Committee of the Third People's Hospital of Jinan (Jinan, China). Written informed consent was obtained from all participants.

General information. A total of 200 patients with T2DM (glycosylated hemoglobin $\geq 7 \%$ ) diagnosed in our hospital (the Third People's Hospital of Jinan) between October 2011 and December 2013 who had previously used OADs, but not insulin, were randomly divided into two groups: Treatment and control. The treatment group included 45 males and 55 females, whereas the control group comprised 47 males and 53 females. The body mass index, and fasting and 2-h postprandial blood glucose, glycosylated hemoglobin, visfatin, APN and blood lipid levels of the patients were tested prior to the treatment. The basic information of the two groups is shown in Table I. 
Table I. Basic information of the two groups.

\begin{tabular}{|c|c|c|c|c|c|c|c|c|c|}
\hline Group & $\mathrm{N}$ & $\begin{array}{c}\text { Age } \\
\text { (years) }\end{array}$ & $\begin{array}{l}\text { Smoking } \\
\text { (years) }\end{array}$ & $\begin{array}{c}\text { Disease } \\
\text { history (years) }\end{array}$ & $\begin{array}{c}\mathrm{TC} \\
(\mathrm{mmol} / \mathrm{l})\end{array}$ & $\begin{array}{c}\mathrm{TG} \\
(\mathrm{mmol} / \mathrm{l})\end{array}$ & $\begin{array}{c}\text { BMI } \\
\left(\mathrm{kg} / \mathrm{m}^{2}\right)\end{array}$ & $\begin{array}{c}\text { FBG } \\
(\mathrm{mmol} / \mathrm{l})\end{array}$ & $\begin{array}{c}\mathrm{PBG} \\
(\mathrm{mmol} / \mathrm{l})\end{array}$ \\
\hline Treatment & 100 & $51 \pm 14.8$ & $6.0 \pm 2.1$ & $5.1 \pm 2.1$ & $6.3 \pm 2.1$ & $1.9 \pm 0.8$ & $24.5 \pm 3.1$ & $8.8 \pm 0.89$ & $12.3 \pm 1.5$ \\
\hline Control & 100 & $52 \pm 14.5^{\mathrm{a}}$ & $6.2 \pm 2.2^{\mathrm{a}}$ & $5.2 \pm 2.0^{\mathrm{a}}$ & $6.2 \pm 2.0^{\mathrm{a}}$ & $1.8 \pm 0.7^{\mathrm{a}}$ & $24.4 \pm 3.2^{\mathrm{a}}$ & $8.7 \pm 0.86^{\mathrm{a}}$ & $12.2 \pm 1.5^{\mathrm{a}}$ \\
\hline
\end{tabular}

Data are presented as the mean \pm standard deviation. ${ }^{\mathrm{a}} \mathrm{P}<0.05$ vs. the treatment group. TC, total cholesterol; $\mathrm{TG}$, triglycerides; $\mathrm{BMI}$, body mass index; FBG, fasting blood glucose; PBG, 2-h postprandial glucose.

Table II. Comparison of the clinical data of the two groups.

\begin{tabular}{|c|c|c|c|c|c|c|c|}
\hline \multirow[b]{2}{*}{ Group } & \multirow[b]{2}{*}{$\mathrm{N}$} & \multicolumn{2}{|c|}{ Glycosylated $\mathrm{Hb}(\%)$} & \multicolumn{2}{|c|}{ APN (mg/l) } & \multicolumn{2}{|c|}{ Visfatin $(\mu \mathrm{g} / \mathrm{l})$} \\
\hline & & Before & After & Before & After & Before & After \\
\hline Control & 100 & $9.3 \pm 1.9$ & $9.2 \pm 1.8^{\mathrm{a}}$ & $0.45 \pm 0.09$ & $0.47 \pm 0.08^{\mathrm{a}}$ & $6.25 \pm 0.6$ & $6.18 \pm 0.5^{\mathrm{a}}$ \\
\hline Treatment & 100 & $9.3 \pm 2.0$ & $8.5 \pm 1.3^{\mathrm{b}}$ & $0.46 \pm 0.08$ & $0.51 \pm 0.14^{\mathrm{b}, \mathrm{c}}$ & $6.24 \pm 0.5$ & $6.17 \pm 0.4^{\mathrm{a}, \mathrm{c}}$ \\
\hline
\end{tabular}

Data are presented as the mean \pm standard deviation. ${ }^{\text {a }}>0.05$ vs. the value before treatment; ${ }^{\text {b }}<0.01$ vs. the value before treatment; ${ }^{\mathrm{c}} \mathrm{P}<0.05 \mathrm{vs}$. the control group; ${ }^{\mathrm{d}}>0.05$ vs. the control group. Hb, hemoglobin; APN, adiponectin.

Treatment. The basal insulin (Ganshulin N, Tonghua Dongbao Pharmaceutical Co., Ltd., Tonghua, China; recombinant insulin glargine Changxiulin, Gan \& Lee Pharmaceutical Co., Ltd., Beijing, China) therapy was administered to the treatment group together with the original OAD (glipizide or metformin). Basal insulin therapy comprised either the use of basal insulin alone or the use of short-acting insulin (Ganshulin R, Tonghua Dongbao Pharmaceutical Co., Ltd.; NovoRapid ${ }^{\circledR}$ Flexpen $^{\circledR}$, Novo Nordisk (China) Pharmaceuticals Co., Ltd., Beijing, China) plus basal insulin (Ganshulin N or recombinant insulin glargine Changxiulin). Basal insulin could be either long- or medium-acting, but pre-mixed insulin was excluded. The patients in the treatment group were instructed to adjust the doses of their drug and insulin intake to enable their fasting and postprandial blood glucose to reach the standards. The control group maintained the original use of OADs (glipizide, Beijing Double-Crane Pharmaceutical Co., Ltd., Beijing, China; metformin, Mogadishu Group Pharmaceutical Co., Ltd., Weihai, China) or used another OAD (carbose, Hangzhou Sino-US East China Pharmaceutical Co., Ltd., Hangzhou, China; pioglitazone, Jiangsu Wanbang Biochemical Pharmaceuticals Co., Ltd., Xuzhou, China) on the above basis to control their fasting and postprandial blood glucose levels within the standard range. Ulnar venous blood was collected for the assessment of the fasting blood glucose, postprandial blood glucose, glycosylated hemoglobin, visfatin and APN levels 12-14 h before treatment. All biochemical parameters were re-examined six months later.

Serum visfatin and APN levels. The enzyme-linked immunosorbent assay (ELISA), with kits from Shanghai Jianglai Chemical Biotechnology Co., Ltd. (Shanghai, China), was used to determine the serum APN and visfatin levels. A 3-5 ml sample of venous blood was obtained, and the supernatant was stored at $-20^{\circ} \mathrm{C}$ subsequent to centrifugation at 2,683 x $\mathrm{g}$ for $5 \mathrm{~min}$. The tests were performed shortly subsequent to the sampling to avoid repeated freezing and thawing. The ELISA was performed in accordance with the manufacturer's instructions (Shanghai Jianglai Chemical Biotechnology Co., Ltd.). The absorbance at a wavelength of $450 \mathrm{~nm}$ was determined using a RT-6500 microplate reader (Shenzhen Leidu Life Sciences Co., Ltd., Shenzhen, China). A standard curve was generated to calculate the concentrations of APN and visfatin.

Glycosylated hemoglobin. The glycosylated hemoglobin levels were measured using high-performance liquid chromatography (Model D-10 ${ }^{\mathrm{TM}}$; Bio-Rad, Hercules, CA, USA), according to the manufacturer's instructions.

Statistical analysis. SPSS 10.0 software (SPSS, Inc., Chicago, IL, USA) was used for the statistical analysis. Data are expressed as the mean \pm standard deviation. The Student's t- test was used for the intergroup comparisons.

\section{Results}

Serum APN. In the treatment group, the comparison of serum APN concentrations before and six months after the basal insulin treatment revealed a significant increase $(\mathrm{P}<0.01)$ (Table II). Furthermore, the serum APN concentration of the treatment group six months after the treatment was significantly higher than that of the control group $(\mathrm{P}<0.05)$. By contrast, the APN concentration of the control group showed no significant difference before and after the treatment $(\mathrm{P}>0.05)$.

Serum visfatin. In the treatment group, the comparison of serum visfatin concentrations before and six months after the basal insulin treatment revealed no change $(\mathrm{P}>0.05)$. In addition, no significant difference was found between the visfatin 
concentrations before and after treatment in the control group ( $\mathrm{P}>0.05$; Table II).

Glyscosylated hemoglobin. In the treatment group, the comparison of glycosylated hemoglobin levels before and six months after the basal insulin treatment revealed a significant decrease $(\mathrm{P}<0.01)$, and the glycosylated hemoglobin concentration of the treatment group six months after the treatment was significantly decreased compared with that of the control group $(\mathrm{P}<0.01)$. In contrast to the treatment group, the control group exhibited no significant change in glycosylated hemoglobin levels before and after the treatment ( $P>0.05$; Table II).

Complications. Two patients in the treatment group and five patients in the control group exhibited hypoglycemia. These patients were relieved of the condition following the prescription of a high-sugar diet.

\section{Discussion}

In China the total prevalence of diabetes is $9.7 \%$, and the prevalence of prediabetes is as high as $15.5 \%$ (22). In patients with T2DM the serum concentration of visfatin is significantly higher than in non-diabetic patients (23). In addition, the APN level is an important predictor for impaired glucose tolerance and T2DM (24). The aims of the present study were to assess the following conditions: i) Whether OAD administration could enable the blood sugar and glycosylated hemoglobin levels of patients with T2DM to return to the standard range; ii) whether the administration of basal insulin would cause changes in visfatin and APN concentrations; and iii) whether a strict control of blood sugar levels could prevent the appearance of diabetic macrovascular complications.

APN is an inflammatory cytokine secreted by fat cells. The gene encoding APN is located at chromosome $3 \mathrm{q} 27$, has a full length of $16 \mathrm{~kb}$ and has been associated with susceptibility to T2DM and metabolic syndrome. A study by Su et al (25) revealed that the APN level was reduced in individuals affected by such metabolic diseases as T2DM and coronary heart diseases. Wong et al (26) found that APN could activate the AMP-activated protein kinase (AMPK)/endothelial nitric oxide synthase (NOS) and cyclic adenosine monophosphate (cAMP)/protein kinase A signaling pathways to increase nitric oxide generation and reduce oxidative stress, thus improving the endothelial functions of mice. Furthermore, Choudhary et al (27) determined that APN could inhibit inflammation through the nuclear factor $\kappa$-light-chain-enhancer of activated B cells $(\mathrm{NF}-\kappa \mathrm{B})$ pathway, thus improving the IR.

A number of studies have confirmed that the association between APN and atherosclerosis can regulate glucose and lipid metabolism and increase insulin sensitivity and fatty acid oxidation. APN is associated with the occurrence and development of T2DM, cardiovascular disease and IR $(28,29)$. It has previously been shown that there is a reduction in the levels of APN before the occurrence of diabetes, in first-degree relatives of patients with T2DM (30). Furthermore, inflammation is closely associated with the pathogenesis of diabetes (31). Inflammatory cytokines and adipokines are involved in the occurrence and development of T2DM and its complications (32). In inflammation, the fibroblasts of the arterial intima proliferate and migrate towards the tunica intima, and then transform into myofibroblasts. The fibroblasts eventually become part of the atherosclerotic plaque. APN can increase the activity of AMPK, inhibit the activity of inducible NOS, reduce the secretion of adventitial inflammatory cytokines and inhibit the proliferation of adventitial fibroblasts and the subsequent transformation into myofibroblasts, ultimately reducing the atherosclerotic plaque area and stabilizing the plaque (33). Under conditions of high glucose, the levels of C-reactive protein in the body are elevated, and the reduced plasma APN then accelerates the diabetic vascular disease and prompts the development of atherosclerosis. A study by Skrabal et al (28) showed that APN caused increases in the plasma free fatty acid oxidation and insulin sensitivity, and regulated glucose and lipid metabolism. Thus, APN can modulate the inflammatory response of endothelial cells and inhibit the transformation of macrophages into foam cells, and is associated with the occurrence and development of obesity, IR, T2DM and cardiovascular diseases.

A prolonged history of T2DM in patients who cannot attain blood glucose standards through the use of OADs can lead do the exhaustion of insular $\beta$-cell functions. Regarding the association between plasma APN and insular $\beta$ cells, a previous study determined (34) that APN was involved in the secretion function of pancreatic $\beta$ cells. APN is a glucose-dependent insulin secretagogue that may, to a certain extent, mediate the inhibition of insulin secretion from pancreatic $\beta$ cells in low-sugar conditions and promote insulin secretion in high-sugar conditions.

The present study revealed that, after six months of basal insulin treatment, the plasma APN concentrations were significantly increased in patients with T2DM who were unable to attain the blood glucose standards (glycosylated hemoglobin $\geq 7 \%$ ). This elevated serum APN could mitigate the endothelial damage and protect the blood vessels from the occurrence of atherosclerosis, thus acting as a compensatory mechanism to benefit the body (35). The results of this study were consistent with the original hypothesis that early basal insulin therapy could increase the plasma concentrations of APN. The analysis of the effects of basal insulin therapy revealed that basal insulin could eliminate the high toxicity of glucose and promote the restoration of insular $\beta$-cell functions, thus increasing the endogenous insulin secretion and promoting the entry of blood glucose into the cells. This would therefore improve the sensitivity of peripheral tissues towards insulin and reduce the IR. Furthermore, the increase in plasma APN would likely prevent the formation of diabetic atherosclerosis.

The generation of visfatin by the visceral fat tissues was higher than that by the subcutaneous fat tissues. Furthermore, the serum visfatin level was increased when the patients with T2DM also presented with macrovascular disease. As such, the association between visfatin and atherosclerosis was investigated. Curat et al (36) revealed that visfatin was primarily released by the macrophages of visceral white adipose tissues, indicating that visfatin was a type of inflammatory cytokine. Dahl et al (37) further suggested that visfatin was a type of inflammatory factor present in macrophages that could play a role in unstable plaques and the injured atherosclerotic vascular endothelium. Diabetes is an inflammatory condition, particularly when the blood glucose control is poor. Under these conditions the serum visfatin concentration is even higher. The increased visfatin could significantly increase the NF- $\mathrm{NB}$ tran- 
scription capabilities inside the endothelial cells and increase the generation of reactive oxygen species, thus resulting in the imbalance of various inflammatory responses. This indicates that visfatin could be involved in various vascular inflammatory processes (38). Kim et al (39) showed that visfatin increased the level of thromboxane synthase, which could consequently promote the production of interleukin- 8 and cause the angiogenesis of endothelial cells. This would lead to the atherosclerosis-induced diabetic microvascular complications.

In the present study it was indicated that visfatin bound to non-insulin binding sites of the insulin receptor when the blood glucose levels of patients with T2DM were increased, thus playing an insulin-like role by inhibiting the release of glycogen, enhancing the glucose uptake by fat and muscle cells and promoting the conversion of glucose into triglycerides, leading to a reduction in the blood sugar level (40). Studies have shown that visfatin can exhibit insulin-like functions, acting in a dose-dependent manner to promote muscle and fat cells to transport glycogen and to inhibit the breakdown of liver glycogen $(41,42)$. Visfatin can also bind to and activate insulin receptors, thus inducing the phosphorylation of the receptors and activating the downstream signal transduction molecules.

The insular $\beta$-cell secretion disorder and IR are two important aspects of T2DM pathogenesis. When a combination of two OADs is unable to control glycosylated hemoglobin levels within the standard range, the pancreatic $\beta$ cells fail and the secretion of endogenous insulin becomes reduced. The body therefore secretes more visfatin to properly control the blood sugar. The visfatin would then induce the phosphorylation of liver insulin receptors and insulin receptor substrate- 2 tyrosine residues, thereby activating protein kinase $\mathrm{B}$ and mitogen-activated protein kinase and the corresponding signaling pathway to reduce the blood sugar levels (43). A previous study also revealed that visfatin has a close association with the progressive failure of insular $\beta$-cell functions. As such, the serum visfatin level would continuously increase with the progressive failure of insular $\beta$-cell functions. Consistent with this, it has been observed that the fasting serum visfatin levels of patients with T2DM are significantly higher than those of non-diabetic subjects (44). It is therefore likely that the increased visfatin levels in patients with diabetes are a compensatory mechanism acting in response to high levels of blood glucose. However, the insulin-like effect of compensatorily increased visfatin is very weak, with poor hypoglycemic function.

In the present study, no change was observed in the serum visfatin concentration before and after six months of basal insulin therapy. The results of this study were inconsistent with the original hypothesis that early basal insulin therapy could increase the plasma concentrations of visfatin. The results revealed that the patients with T2DM that could not be controlled by OAD suffered from impaired insular $\beta$-cell functions. Thus, the endogenous insulin secretion was decreased, as the plasma visfatin had the insulin-like functions of binding the insulin receptor and promoting the blood sugar to enter the cells, consequently causing a blood sugar reduction. When the basal insulin therapy was administered, the exogenous insulin bound to the insulin receptors. This prevented the serum visfatin from binding to the other sites of the insulin receptor available under conditions of proper blood glucose control. Accordingly, the serum visfatin concentrations increased. The finding that the serum visfatin did not exhibit a significant reduction indicated that the islet $\beta$ cells did not completely fail. A certain amount of visfatin, which combined with the insulin receptors due to the existence of endogenous insulin, still existed. Thus, the serum concentrations of free visfatin increased.

The 10-year follow-up results of the United Kingdom Prospective Diabetes Study suggested that actively reducing blood sugar and maintaining long-term good blood glucose control from the early stages of diabetes could be beneficial in the establishment of an effective metabolic memory, thus preventing or delaying the occurrence and development of chronic diabetes-related complications (45). Basal insulin supplements could reduce the fasting blood glucose and hepatic glucose output in patients with diabetes. The insular functions could be protected and the failure of $\beta$-cell functions could be delayed by controlling the blood sugar levels via basal insulin (46). In this event, the glycosylated hemoglobin and postprandial glucose levels would also be maintained within the standard range. It was found in the present study that the patients with T2DM, who could not achieve satisfactory results with OAD administration, could attain a significant reduction in glycosylated hemoglobin after a six-month basal insulin therapy $(\mathrm{P}<0.01)$. The control group were treated with OAD only, and although the glycosylated hemoglobin level decreased, this difference was not significant.

This study indicated that the administration of basal insulin therapy was beneficial for the patients with T2DM who were not able to achieve satisfactory results with OAD therapy (glycosylated hemoglobin $\geq 7 \%$ ). Such medication could return the blood glucose levels to within the standard range and increase the serum APN levels. This could thus control diabetic microvascular complications and prevent diabetic macrovascular complications. These results were consistent with the findings of other studies. In the clinical application, the incidence of hypoglycemia with basal insulin administration was relatively lower than that with OAD administration. The serum visfatin of the basal insulin group did not show a significant decrease, which was inconsistent with the findings of previous studies; this may have been due to the smaller sample size of the present study or because the application of basal insulin was too late or the observation period was too short.

\section{Acknowledgements}

This study was supported by the Science and Technology Project of Jinan Municipal Health Bureau.

\section{References}

1. Robetson RP, Harmon J, Tran PO, Tanaka Y and Takahashi H: Glucose toxicity in beta-cells: type 2 diabetes, good radicals gone bad, and the glutathi-one connection. Diabetes 52: 581-587, 2003.

2. Cobitz AR and Ambery P: Medical management of hyperglycemia in type 2 type diabetes: a consensus algorithm for the initiation and adjustment of therapy: a consensus statement of the American Diabetes Association and the European Association for the Study of Diabetes. Response to Nathan et al. Diabetes Care 32: e58, 2009.

3. Swinnen SG, Dain MP, Aronson R, et al: A 24-week, randomized, treat-to-target trial comparing initiation of insulin glargine once-daily with insulin detemir twice-daily in patients with type 2 diabetes inadequately controlled on oral glucose-lowering drugs. Diabetes Care 33: 1176-1178, 2010. 
4. Riddle MC, Rosenstock J, Gerich J; Insulin Glargine 4002 Study Investigators: The treat-to-target trial: Randomized addition of glargine or human NPH insulin to oral therapy of type 2 diabetic patients. Diabetes Care 26: 3080-3086, 2003.

5. Janka HU, Plewe G, Riddle MC, et al: Comparison of basal insulin added to oral agents versus twice-daily premixed insulin as initial insulin therapy for type 2 diabetes. Diabetes Care 28: 254-259, 2005.

6. Yki-Järvinen H, Kauppinen-Mäkelin R,Tiikkainen M, et al: Insulin glargine or NPH combined with metformin in type 2 diabetes: The LANMET study. Diabetologia 49: 442-451, 2006.

7. Lepore M, Pampanelli S, Fanelli C, et al: Pharmacokinetics and pharmacodynamics of subcutaneous injection of long-acting human insulin analog glargine, NPH insulin, and ultralente human insulin and continuous subcutaneous infusion of insulin lispro. Diabetes 49: 2142-2148, 2000

8. Stentz FB, Umpierrez GE, Cuervo $R$ and Kitabchi AE: Proinflammatory cyto-kines, markers of cardiovascular risks, oxidative stress, and lip id peroxide-tion in patients with hyperglycemic crises. Diabetes 53: 2079-2086, 2004.

9. Forouhi NG, Sattar N and McKeigue PM: Relation of C-reactive protein to body fat distribution and features of the metabolic syndrome in Europeans and South Asians. Int J Obes Relat Metab Disord 25: 1327-1331, 2001

10. Nayak BS, Ramsingh D, Gooding S, et al: Plasma adiponectin levels are related to obesity, inflammation, blood lipids and insulin in type 2 diabetic and non-diabetic Trinidadians. Prim Care Diabetes 4: 187-192, 2010.

11. Winkler C and Cseh K: Molecular mechanisms and correlations of insulin resistance, obesity, and type 2 diabetes mellitus. Orv Hetil 150: 771-780, 2009 (In Hungarian).

12. Inoue M, Maehata E, Yano M, Taniyama M and Suzuki S: Correlation between the adiponectin-leptin ratio and parameters of insulin resistance in patients with type 2 diabetes. Metabolism 54 281-286, 2005

13. Lindsav RS, Funabashi T, Hanson RL, et al: Adiponectin and development of type 2 diabetes in the Pima Indian population. Lancet 360: 57-58, 2002

14. Kubota N, Terauchi Y, Yamauchi T, et al: Disruption of adiponectin causes insulin resistance and neointimal formation. J Bio Chem 277: 25863-25866, 2002

15. Stefan N, Stumvoll M, Vozarova B, et al: Plasma adiponectin and endogenous glucose production in humans. Diabetes Care 26 3315-3319, 2003

16. Hivert MF, Sullivan LM, Shrader P, et al: Insulin resistance influences the association of adiponectin levels with diabetes incidence in two population-based cohorts: the Cooperative Health research in the Region of Augsburg (KORA) S4/F4 study and the Framingham Offspring Study. Diabetologia 54: 1019-1024, 2011.

17. Lau CH and Muniandy S: Adiponectin and resistin gene polymorphisms in association with their respective adipokine levels. Ann Hum Genet 75: 370-382, 2011.

18. Chu CH, Lam HC, Lee JK, et al: Hyperthyroidism-associated insulin resistance is not mediated by adiponectin levels. J Thyroid Res 2011: 194721, 2011.

19. Wolf AM, Wolf D, Rumpold H, Enrich B and Tilg H: Adiponectin induces the anti-inflammatory cytokines IL-10 and IL-1RA in human leukocytes. Biochem Biophys Res Commun 323: 630-635, 2004.

20. Imai S: Nicotinamide phosphoribosyltansferase (Nampt): a link between NAD biology, metabolism, and diseases. Curr Pharm Des 15: 20-28, 2009.

21. Lin $\mathrm{Y}$, Berg $\mathrm{AH}$, Iyengar $\mathrm{P}$, et al: The hyperglycemia-induced inflammatory response in adipocytes: the role of reactive oxygen species. J Biol Chem 280: 4617-4626, 2005

22. Yang W, Lu J, Weng J, et al: Prevalence of diabetes among men and women in China. N Engl J Med 362: 1090-1101, 2010.

23. Chen MP, Chung FM, Chang DM, et al: Elevated plasma level of vifatin/pre-B cell colony-enhancing factor in patients with type 2 diabetes mellitus. J Clin Endocrinol Metab 91: 295-299, 2006

24. Jalovaara K, Santaniemi M, Timonen M, et al: Low serum adiponectin level as a predictor of impaired glucose regulation and type 2 diabetes mellitus in a middle-aged Finnish population. Metabolism 57: 1130-1134, 2008.
25. Su SC, Pei D, Hsieh CH, et al: Circulating pro-inflammatory cytokines and adiponectin in young men with type 2 diabetes. Acta Diabetol 48: 113-119, 2011.

26. Wong WT, Tian XY, Xu A, et al: Adiponectin is required for PPAR $\gamma$-mediated improvement of endothelial function in diabetic mice. Cell Metab 14: 104-105, 2011.

27. Choudhary S, Sinha S, Zhao Y, et al: NF-kappaB-inducing kinase (NIK) mediates skeletal muscle insulin resistance: blockade by adiponectin. Endocrinology 152: 3622-3627, 2011.

28. Skrabal CA, Czaja J, Honz K, et al: Adiponectin - its potential to predict and prevent coronary artery disease. Thorac Cardiovasc Surg 59: 201-206, 2011.

29. Kyriazi E, Tsiotra PC, Boutati E, et al: Effects of adiponectin in TNF- $\alpha$, IL-6, IL-10 cytokine production from coronary artery disease macrophages. Horm Metab Res 43: 537-544, 2011.

30. Tesauro M, Rizza S, Iantorno M, et al: Vascular, metabolic, and inflammatory abnormalities in normoglycemic offspring of patients with type 2 diabetes mellitus. Metabolism 56: 413-419, 2007.

31. Pickup JC: Inflammation and activated innate immunity in the pathogenesis of type 2 diabetes. Diabetes Care 27: 813-823, 2004

32. Donath MY and Shoelson SE: Type 2 diabetes as an inflammatory disease. Nat Rev Immunol 11: 98-107, 2011.

33. Cai XJ, Chen L, Li L, et al: Adiponectin inhibits lipopolysaccharide-induced adventitial fibroblast migration and transition to myofibroblasts via AdipoR1-AMPK-iNOS pathway. Mol Endocrinol 24: 218-228, 2010.

34. Fagerberg B, Kellis D, Bergström G and Behre CJ: Adiponectin in relation to insulin sensitivity and insulin secretion in the development of type 2 diabetes: a prospective study in 64-year-old women. J Intern Med 269: 636-643, 2011.

35. Ran J, Xiong X, Liu W, et al: Increased plasma adiponectin closely associates with vascular endothelial dysfunction in type 2 diabetic patients with diabetic nephropathy. Diabetes Res Clin Pract 88: 177-183, 2010

36. Curat CA, Wegner V, Sengenès C, et al: Macrophages in human visceral adipose tissue: increased accumulation in obesity and a source of resistin and visfatin. Diabetologia 49: 744-747, 2006.

37. Dahl TB, Yndestad A, Skjelland M, et al: Increased expression of visfatin in macrophages of human unstable carotid and coronary atherosclerosis: possible role in inflammation and plaque destabilization. Circulation 115: 972-980, 2007.

38. Adya R, Tan BK, Chen J and Randeva HS: Nuclear factor-kappaB induction by visfatin in human vascular endothelial ceils: its role in MMP-2/9 production and activation. Diabetes Care 31: 758-760, 2008

39. Kim SR, Jung YH, Park HJ, et al: Upregulation of thromboxane synthase mediates visfatin-induced interleukin-8 expression and angiogenic activity in endothelial cells. Biochem Biophys Res Commun 418: 662-668, 2012.

40. Kadoglou NP, Sailer N, Moumtzouoglou A, et al: Visfatin (nampt) and ghrelin as novel markers of carotid atherosclerosis in patients with type 2 diabetes. Exp Clin Endocrinol Diabetes 118 75-80, 2010.

41. Sethi JK and Vidal-Puig A: Visfatin: the missing link between intra-abdominal obesity and diabetes? Trends Mol Med 11: 344-347, 2005 .

42. Samal B, Sun Y, Stearns G, Xie C, Suggs S and McNiece I: Cloning and characterization of the cDNA encoding a novel human pre-B-cell colony-enhancing factor. Mol Cell Biol 14: 1431-1437, 1994

43. Hug C and Lodish HF: Medicine. Visfatin: a new adipokine. Science 307: 366-367, 2005 .

44. Retnakaran R, Youn BS, Liu Y, et al: Correlation of circulating full-length visfatin (PBEF/NAMPT) with metabolic parameters in subjects with and without diabetes: a cross-sectional study. Clin Endocrinol (Oxf) 69: 885-893, 2008.

45. Holman RR, Paul SK, Bethel MA, Matthews DR and Neil HA: 10-year follow-up of intensive glucose control in 2 type diabetes. N Engl J Med 359: 1577-1589, 2008

46. Forst T, Larbig M, Hobberg C, et al: Adding insulin glargine vs. NPH insulin to metformin results in a more efficient postprandial beta-cell protection in individuals with type 2 diabetes. Diabetes Obes Metab 12: 437-441, 2010. 